\title{
A Generalization of the Morse Index Theorem to a Class of Degenerate Elliptic Operators
}

\author{
L. M. SIBNER \\ Communicated by F. W. GeHring
}

In a recent paper, J. J. Kohn and L. Nirenberg show that some of the fundamental properties of strongly elliptic operators also hold for a class of degenerate elliptic operators (see [1]). A simple example which is typical of some of those they consider is the second order operator in the plane having principal part

$$
y^{2} \frac{\partial^{2}}{\partial x^{2}}+\frac{\partial^{2}}{\partial y^{2}}
$$

which is elliptic except on the real axis.

The purpose of this paper is to show that the classical Sturm-Liouville theory is valid for problems satisfying the Kohn-Nirenberg conditions. In particular, the proof of the Morse Index Theorem for strongly elliptic operators due to Smale (see [2]) can be modified for an appropriate class of degenerate problems.

We consider vector valued functions $u=\left(u_{1}, \cdots, u_{p}\right)$ defined in the closure of a bounded open set $M$ in $R^{n}$ having smooth boundary. Let $B$ be the subspace of $\mathfrak{e}^{\infty}(\bar{M})$ consisting of vector-valued functions which vanish up to order $k-1$ on $\partial M$. We will be concerned with linear differential operators $L$ of order $2 k$ defined on $B$. For every $u, v \varepsilon B$, the $L_{2}$ inner product $(L u, v)$ defines a bilinear form $Q(u, v)$ in the derivatives of $u$ and $v$ up to order $k$; i.e.,

$$
(L u, v)=Q(u, v)=\int_{M} \sum_{i, i} \sum_{\substack{\alpha|\leqq k\\| \beta \mid \leqq k}} a_{i i}^{\alpha \beta} D^{\alpha} u^{i} \overline{D^{\beta} v^{j}} d x
$$

with smooth matrix valued coefficients. $L$ is called self-adjoint if $Q(u, v)$ is Hermitian.

The Morse Index $I(L)$ is then defined as the dimension of the largest subspace of $B$ on which the quadratic form $Q(u, u)$ is Hermitian negative definite.

A smooth deformation of $M$ is a family of domains $M_{t}, 0 \leqq t \leqq 1$, such that $M_{0}=M$ and $M_{t}$ is properly contained in $M_{s}$ for $t>s$. Moreover, the boundary of $M_{t}$ depends on $t$ in a smooth way. Such a deformation is of $\epsilon$-type if the measure of $M_{1}$ is smaller than $\epsilon$. We will always assume that for every $\epsilon>0$, 
$M$ is such that a smooth deformation of $\epsilon$-type exists. For a given deformation $M_{t}$, let $L_{t}: B\left(\bar{M}_{t}\right) \rightarrow \mathcal{C}^{\infty}\left(\bar{M}_{t}\right)$ be the restriction of $L$ to $B\left(\bar{M}_{t}\right)$. The number $t_{0}, 0<t_{0}<1$, is called a focal point if there is a function $\varphi \varepsilon B\left(\bar{M}_{t_{0}}\right)$ not identically zero satisfying $L_{t_{0}} \varphi=0$. The multiplicity $m\left(t_{0}\right)$ is defined as the dimension of the space of solutions of the above homogeneous problem.

The Morse Index Theorem then states that under suitable hypotheses on $L$, if $M_{t}$ is of $\epsilon$-type for sufficiently small $\epsilon$,

$$
I(L)=\sum_{0<t<1} m(t)<\infty .
$$

The required hypotheses may be formulated as three conditions on the operators $L_{t}$. Namely,

(i) For every $t, 0 \leqq t \leqq 1, L_{t}$ has a non-decreasing sequence $\lambda_{n}^{t}$ of eigenvalues, such that $\lambda_{n}^{t} \rightarrow \infty$ as $n \rightarrow \infty$, and for each $n$ the associated eigenspace has finite dimension.

(ii) Let $\lambda_{n}^{t}$ be the $n^{t h}$ eigenvalue of $L_{t}$. For each $n, \lambda_{n}^{t}$ is a continuous, strictly increasing function of $t$.

(iii) For every $N>0$, there is an $\epsilon>0$ depending on $N$, such that if the measure $M_{t}$ is smaller than $\epsilon$, all eigenvalues $\lambda_{n}^{t}$ of $L_{t}$ satisfy: $\left|\lambda_{n}^{t}\right| \geqq N$. It follows from (i) that $L$ has at most a finite number of negative eigenvalues, each counted according to their multiplicity, say $s$. Clearly, $I(L)=s$. From (i), (ii) and (iii), it follows that if $\epsilon$ is sufficiently small than all eigenvalues of $L_{1}$ are strictly greater than zero. Taking the dependence of the $n^{\text {th }}$ eigenvalue on $t$ into account, it is clear that (1) holds. Hence,

Theorem 1. Let $L$ be a self-adjoint operator defined on $B(\bar{M})$ and satisfying (i), (ii) and (iii). Then, there is an $\epsilon>0$ and a smooth deformation of $\epsilon$-type such that (1) holds.

The theorem is due to Smale in the case where $L$ is strongly elliptic and has the "weak unique continuation" property. Under these conditions, (i), (ii) and (iii) are satisfied.

Next we define the class of degenerate elliptic operators. We always assume that $L$ has smooth coefficients and that the boundary of $M$ is smooth.

Let $H_{m}$ be the completion of $\mathcal{e}_{\infty}(\bar{M})$ with respect to the norm

$$
\|u\|_{m}^{2}=\sum_{|\alpha| \leqq m} \int_{M}\left|D^{\alpha} u\right|^{2} d x \quad m=0,1, \cdots
$$

and denote by $(,)_{m}$ the inner product in $H_{m}$.

We always assume that

$$
(L u, v)=Q_{0}(u, v)-c(u, v)_{k-1}
$$

where $c$ is a positive constant, $Q_{0}$ is Hermitian and

$$
Q_{0}(u, u) \geqq\|u\|_{k-1}^{2} \text {. }
$$

This defines a self-adjoint operator $L_{0}$ satisfying 


$$
\left(L_{0} u, v\right)=Q_{0}(u, v) \text { for } u, v \varepsilon B .
$$

The lower bound on $Q_{0}(u, u)$ insures that for every complex vector $v=$ $\left(v^{\prime}, \cdots, v^{p}\right)$

$$
\sum_{i, i} \sum_{|\alpha|=|\beta|=k} a_{i j}^{\alpha \beta} v_{i} \bar{v}_{i} \geqq 0 .
$$

Let $\mathfrak{K}$ be the completion of $B$ with respect to the norm $Q_{0}(u, u)^{1 / 2}$. The class of operators which we consider may then be characterized by the following two properties:

(a) The unit ball in $\mathfrak{K}$ is conditionally compact in $H_{k-1}$.

(b) The boundary of $M$ is nowhere characteristic for $L$; i.e., at any boundary point of $M$, if $\xi$ is a vector normal to the boundary then

$$
\sum_{|\alpha|+|\beta|=2 k} a_{i j}^{\alpha \beta} \xi^{\alpha+\beta}
$$

is non-singular. Conditions (a) and (b) are always satisfied if $L$ is strongly elliptic, but they do not imply strong ellipticity. For example, these conditions are met by the form

$$
Q_{0}(u, v)=\iint_{M}\left(y^{2} u_{x} v_{x}+u_{y} v_{y}+u v\right) d x d y
$$

where $M$ is any bounded open set whose boundary is smooth and does not intersect the $x$ axis orthogonally.

The main theorem of Kohn-Nirenberg which we shall use is the following:

Theorem 2. Let $L$ and $M$ be such that (a) and (b) hold. Then,

(i) For every $f \boldsymbol{\varepsilon} \mathcal{C}^{\infty}(\bar{M})$, the equation

$$
Q_{0}(u, v)=(f, v)_{0} \quad \text { for every } \quad v \varepsilon B
$$

has a unique solution $u \varepsilon B$. Moreover, the mapping $L_{0}^{-1}: f \rightarrow u$ is compact in each $H_{m}, m \geqq k-1$.

(ii) For every $\delta>0$, there is a constant $C=C(\delta)$, such that for $u \varepsilon B$,

$$
\|u\|_{k}^{2} \leqq \delta^{2}\left\|L_{0} u\right\|_{k}^{2}+C(\delta)\|u\|_{k-1}^{2} .
$$

The proof of Theorem 2 may be found in [1].

The results of this theorem together with the Fredholm-Riesz theory for compact operators imply that $L$ has a non-decreasing sequence of eigenvalues tending to $+\infty$, that the eigenspace of each eigenvalue has finite dimension, that the eigenfunctions are in $\mathfrak{e}^{\infty}$, and that they form a complete orthonormal set in $H_{k-1}$. If moreover, we assume

(c) For every $\epsilon>0, M$ is such that there is a smooth deformation of $\epsilon$-type such that for each $t, 0 \leqq t \leqq 1$, the boundary of $M_{t}$ is nowhere characteristic for $L_{t}$.

Then these results hold for every $L_{t}$. Hence, for operators $L$ satisfying (a), (b), (c), condition (i) holds.

In the strongly elliptic case, condition (iii) is a consequence of Gärding's 
inequality. We now prove (iii) using the weaker inequality of Theorem 2. Choose $N>0$. Let $\delta>0$ be so small that $1 / 2 \delta^{2}>N^{2}$. This then determines the constant $C(\delta)$ in Theorem 2 . Next, let $\gamma$ be any positive number satisfying: $C(\delta) \gamma<\frac{1}{2}$. By a well known inequality, if the measure of $M_{t}$ is sufficiently small, then,

It follows that

$$
\|u\|_{k-1}^{2} \leqq \gamma\|u\|_{k}^{2} \quad \text { for } \quad u \varepsilon \mathcal{C}_{0}^{\infty}\left(\bar{M}_{t}\right)
$$

$$
\|u\|_{k}^{2} \leqq 2 \delta^{2}\left\|L_{0}^{t} u\right\|_{k}^{2} \quad \text { for } \quad u \varepsilon \mathbb{C}_{0}^{\infty}\left(\bar{M}_{t}\right) .
$$

Setting $L_{0}^{t} u=\lambda_{0}^{t} u$, it follows that $\left|\lambda_{0}^{t}\right| \geqq N$. Since the eigenvalues of $L_{t}$ are related to those of $L^{t}$ by a translation, (iii) is satisfied.

The proof of (ii) proceeds exactly as in the strongly elliptic case (see [2]). One shows that $\lambda_{n}^{t}$ is a continuous increasing function of $t$ from the variational formula for the $n^{\text {th }}$ eigenvalue of a compact self-adjoint operator (see Courant \& Hilbert [3]). Namely,

$$
\lambda_{n}=\max _{\operatorname{dim} V<n} \min _{\rho \varepsilon \delta \cap V^{\perp}} Q_{(\rho, \rho)}=\min _{\operatorname{dim} V \geq n} \max _{\rho \varepsilon} Q_{(\rho, \rho)}
$$

where $V$ is any subspace of $H_{k}$ and $\subseteq$ is the unit sphere in $H_{k-1}$.

To obtain strict monotonicity, one needs to assume

(d) $L$ has the "weak unique continuation" property; $u=0, L u=0$ in an open set implies $u \equiv 0$. The proof of this may be found in Smale's paper.

In conclusion, we have shown

Theorem 3. Let $L$ be a self-adjoint operator and assume that (a), (b), (c) and (d) hold in the bounded domain $M$. Then, for $\epsilon>0$ sufficiently small, if $M_{\text {。 }}$ is a smooth non-characteristic deformation of $\epsilon$-type, then

$$
I(L)=\sum_{0<t<1} m(t)<\infty .
$$

Note that the above theorem is applicable to the degenerate equation

$$
L u=y^{2} u_{x x}+u_{y y}+\rho(x, y) u
$$

defined in any bounded non-characteristic domain having smooth boundary.

The author wishes to thank Professor L. Nirenberg for many invaluable discussions.

\section{BibliogRAPHY}

[1] J. J. Kohn \& L. Nirenberg, Non-coercive boundary value problems, Comm. Pure Appl. Math, 18 (1965) 443-492.

[2] S. Smale, On the Morse Index Theorem, J. Math. Mech., 14 (1965) 1049-1055.

[3] R. Courant \& D. Hilbert, Methods of Math Phys., Vol. 1, New York, 1953, Chapter VI.

Polytechnic Institute of Brooklyn

Brooklyn, New York

Date communicated: ОстовER 30, 1968 